\title{
News on Silicon Drift Detectors for X-Ray Nanoanalysis in S/TEM
}

\author{
M. Falke, ${ }^{*}$ R. Kroemer, ${ }^{*}$ D. Fissler, $*$ and M. Rohde* \\ * Bruker-AXS Microanalysis GmbH, Berlin, Schwarzschildstr. 12, Germany, 12489
}

Silicon Drift Detectors (SDDs) for X-ray analysis, which were initially introduced for space research and later utilized [1] very successfully for energy dispersive X-ray spectroscopy (EDS) in Scanning Electron Microscopy (SEM) have recently made their way into transmission electron microscopy as well. For a few years now SDD-EDS has been used for nanoanalysis in S/TEM. We will report on the respective latest developments, results and progress of SDD technology in S/TEM.

Electron transparent samples provide less signal compared to bulk. Thus, to get as many X-ray counts as possible by covering a large solid angle belongs to the major challenges for SDDdevelopment in STEM. The improvement of the count rate can be supported by high brightness sources and spherical (Cs) and chromatic (Cc) aberration correction, which help to provide a high current in an electron probe of nm or Angstrom diameter. Unfortunately, beam sensitivity of the sample counteracts these measures and requests a very fast data acquisition, again possible only with a large solid angle for signal detection. This being not enough, the best possible EDS data interpretation is possible only with a good P/B-ratio, which depends on the take off angle. The most valuable EDS data is produced only if the highest possible take off angle is achieved, which further complicates the geometrical problem of fitting the detector into the TEM pole piece region.

Another important point is that the detector technology used should not compromise any of the microscope specifications, also in case of aberration corrected instruments placed in special expensive low noise environments.

Small and medium size detector areas provide higher throughput and need less cooling compared to large detector areas, which is important to enable stable measurement conditions. Additionally, because of geometric constraints, small detectors can be placed more easily close to the sample thus enabling a large solid angle and a good take off angle as well. Both, respective single and multidetector solutions proved to be a suitable way to meet all the requests for EDS nanoanalysis.

Fig. 1. shows an 8 minute mapping of a $\mathrm{NiSi}_{2} / \mathrm{Ni}$ film on Silicon (001) [2], with a content of nominally 5 at\% of Platinum. Platinum, added to stabilize the desired NiSi-Phase, is soluble only in the Ni monosolicide and not in the disilicide phase, which is clearly reproduced. Pt is difficult to detect reliably using EELS. An SDD area of $30 \mathrm{~mm}^{2}$ covering a solid angle of $0.12 \mathrm{sr}$ at a take off angle of $22.5^{\circ}$ was used with the sample not tilted. The spectra on the right derived from the sample areas as marked in the map confirm the Pt distribution in the monosilicide.

Fig. 2. demonstrates, that within one minute the Co catalyst within a multiwall carbon nanotube (MWCNT) [2] can be identified under the same geometrical conditions as described above. It is clearly distinguishable from the $\mathrm{Cu}$-particles of the tube coating which appear bright in Z-contrast in HAADF as well. The MWCNT $\mathrm{Cu}$ coating has been confirmed by EELS in a Cs-corrected instrument at $80 \mathrm{kV}$ accelerating voltage. 
This data shows that improved detectors and multi-detector systems will provide the means to map even smaller amounts of matter during shorter acquisition times. Thus, routine atomic resolution EDS, in-situ EDS and 3-D-EDS in S/TEM is within reach.

\section{References}

[1] Strüder, et al., Microsc Microanal 4 (1999), 622.

[2] The samples were prepared at Chemnitz University of Technology, Germany
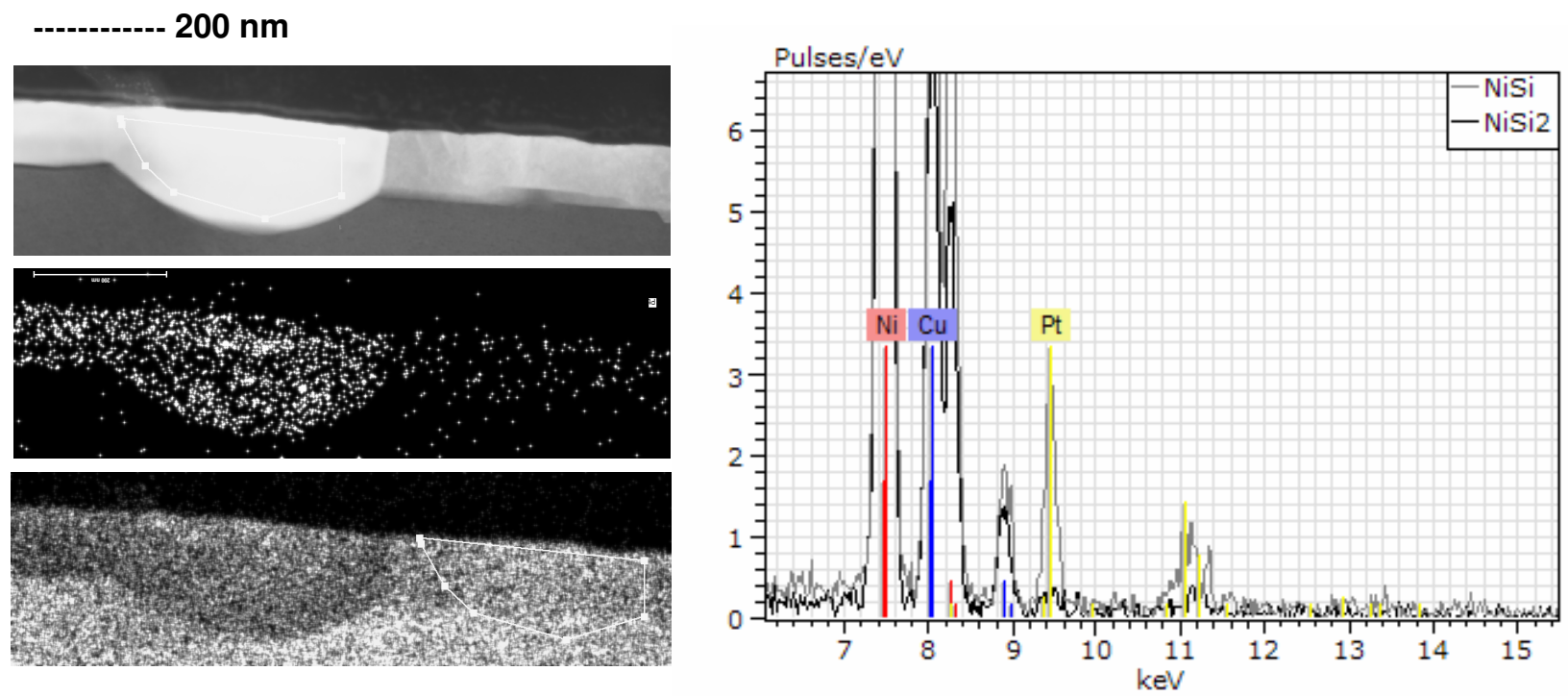

FIG.1. 8 min map of a NiSi / NiSi 2 film on $\mathrm{Si}$ (001). HAADF (top left), Pt-map (middle left), Si-map (lower left) and on the right: spectra in the $\mathrm{NiSi}$ and $\mathrm{NiSi}_{2}$ regions as marked, scale bar: $200 \mathrm{~nm}$. The thickness of the $\mathrm{NiSi}_{2}$-film on the right is $60 \mathrm{~nm}$. The heavier NiSi (left) appears bright in Z-contrast in the high angle annular dark field image. The element map shows clearly, that the Platinum is distributed in the monosilicide. The thin bright line on top of the layer is caused by a thin $2 \mathrm{~nm}$ band of $\mathrm{Cu}$, deposited during ion milling.

------ $30 \mathrm{~nm}$
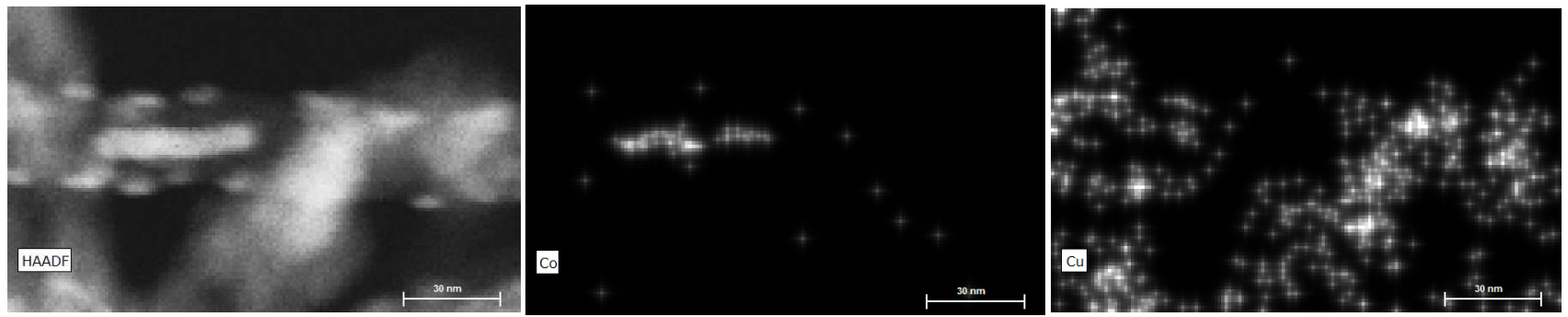

FIG. 2. EDS of multiwall carbon nanotubes on a lacy carbon gold grid. On the left: HAADF, Zcontrast; middle: Co-map; right: $\mathrm{Cu}$-map. The Co-catalyst particle, few $\mathrm{nm}$ in diameter, was clearly revealed during this 1 min EDS-mapping. The scale bar is $30 \mathrm{~nm}$. 